elSSN: 09748369, www.biolmedonline.com

\title{
Stress induced by beta-cyfluthrin, a type-2 pyrethroid, on brain biochemistry of Albino rat (Rattus norvegicus)
}

\author{
AK Singh*, PN Saxena, HN Sharma \\ Toxicology Laboratory, Department of Zoology, School of Life Sciences, Khandari Campus, \\ Dr. B.R. Ambedkar University, Agra-282002 (U.P.), India. \\ *Corresponding author: aks_8480@yahoo.co.in
}

\begin{abstract}
Beta-Cyfluthrin is one of the most widely used type-II pyrethroid in agriculture. Toxic effects of beta-cyfluthrin are due to the presence of cyano moiety in its chemical structure. This study was designed the effect of betacyfluthrin at different doses on brain tissue, the most important vital organ in animal body and in which dose level beta-cyfluthrin shows its effects. Animal were initially divided into control and beta-cyfluthrin given groups. There were five animals in the control group and twenty five animals in beta-cyfluthrin administered group. The latter was divided into five equal subgroups: 35.48 , and 5.06, 2.53, 1.68, 1.27mg/kg body weight of beta-cyfluthrin administered groups, acute (1 day) and sub-acute (7, 14, 21 and 28 days) by gavage respectively. Control group was given only water. The effect of these treatments were studied on activities of four potential biomarkers were assayed in the brain of albino rat along with neurosomatic index viz. Body weight, brain weight and brain weight body weight ratio and neurobehavioural changes. Beta-cyfluthrin treatment resulted in a significant decrease in brain acetyl-cholinesterase. Acetylcholinesterase which found to be decreased (inhibition range 53 to 18\%) in albino rats after acute and sub-acute treatment (vide-supra). Further glutathione-s-transferase (GST) was also found to be decreased in brain of albino rats (inhibition range 47 to29\%) after beta-cyfluthrin administration. Again brain adenosine triphosphtase (total ATPase) activity was seen to decrease in albino rats (inhibition range 36 to $19 \%$ ) along with succinic dehydrogenase (SDH) was also seen decreased in brain of albino rats (inhibition range 31 to $9 \%$ )) after acute and sub-acute beta-cyfluthrin intoxication. Further hypnoatremia (inhibition range 31 to $20 \%$ ) along with hypokalemia (inhibition range 19 to $14 \%$ ) has also been observed after acute and sub-acute intoxication of beta-cyfluthrin. Apart from AChE, which is a specific marker of pyrethroid neurotoxicity, the levels of brain GST, ATPase, SDH along with $\mathrm{Na}$ and $\mathrm{K}$ may serve as important determinants of beta-cyfluthrin induced neuronal dysfunctioning along with neurobehavioural alterations; outcomes of fluctuated brain biochemistry because clinical signs of toxicity were observed in animals which received different doses of beta-cyfluthrin after $7^{\text {th }}$, and $14^{\text {th }}$ days of sub-acute treatment. Animals showed overt cholinergic signs which included salivation, excitability, ataxia, muscle twisting, followed by general tremors and lethargyness. The treated groups did not reveal any mortality. Exposure of animals to beta-cyfluthrin caused extensive changes in neurosomatic, neurochemical and neurobehavioural parameters. So, oral administration of beta-cyfluthrin leads to negative response on animal health.
\end{abstract}

Keywords: Beta-cyfluthrin; Biochemical markers; Brain; Behavioural parameters; Rat.

\section{Introduction}

Brain is the most important vital organ in animal body as it is a site of controlling and coordinating with other vital organs and their respective functions. It is always active in both active and steady state of the animal, however, its activity rhythms can be altered with a slight change in the external environment as well as in the internal milieu. A foreign body in form of a chemical stress is sufficient enough to give jolt either mild or severe in its functioning potential. Studies have been conducted on plethora of synthesized chemicals from past to present. However, some of the synthesized chemicals have received attention as they were considered to be safe for mammalian system. A tendency of repeated exposure to different animal system of the food chain has always intervened ongoing metabolic activities, to some extent. This extent could be magnified for the safe listed chemicals if their application is not restricted, before it gets too late. Synthetic pyrethroids are such synthesized chemicals, overwhelmingly accepted throughout the globe in farm practices for different categories of insect pests. They induce almost similar system like the ones of their fore generations (the first synthetic OCls). Beta-cyfluthrin of the pyrethroid series, a safe chemical of yester years has banged for its presence in the vital organs. It is with this reason an effort has been phased out to observe changes at the level of brain as it has all centres for controlling physiological activities going on in the body. Beta-cyfluthrin, (SR)-alpha-cyano-4-fluoro-3-= phenoxybenzyl (1RS, 3RS; 1RS, =3SR)-3-(2,2 -dichlorovinyl)- 
2,2 - = dimethylcyclopropane carboxylate, being a type-II pyrethroid has potential use as an ectoparasiticide in animals. It is highly toxic to fish, aquatic investigators with less toxicity to higher non-target mammals. It degrades in sun light, can be metabolized easily and also depicts other typical type II characteristics. Beta-cyfluthrin is a potent neurotoxin, with a mode of action similar to that of the organochlorine insecticide DDT (Cox, 1994). The introduction of cyano group enhances neurotoxicity in mammals. It may enter animal or human system either directly or indirectly as an environmental pollutant. Hence, the commercially available formulation of Betacyfluthrin has been selected for the present investigation. The main objective of this study was to find out whether beta-cyfluthrin (in low and high concentrations) has a significant effect on some potential biomarkers which has been adjudged on the basis of neurosomatic, neurobehavioural and neurochemical responses.

\section{Materials and Methods}

\section{Rearing of experimental animal}

The experiment was conducted on acclimated albino rats, weighing $120 \pm 10 \mathrm{gms}$, from inbred colony, maintained at temperature $25 \pm 5^{\circ} \mathrm{C}$, relative humidity $60 \pm 5 \%$ and photoperiod of 10 hours per day. All the animals were allowed free access to standard rodent pellet diet obtained from Hindustan Antibiotic Ltd., India and water was provided ad libitum.

\section{Chemical formulations and $L D_{50}$ determination}

Commercial formulation beta-cyfluthrin (Bulldock 025SC) obtained from Bayer India Ltd., New Delhi, was in the form of emulsion and adequate dilutions were done in water to prepare various test concentrations of betacyfluthrin. Freshly prepared solution of betacyfluthrin in water in five different concentrations (100, 200, 400, 800 and 1600 $\mathrm{mg} / \mathrm{kg}$ body weight) were given to five groups, each containing seven individuals of albino rat, orally by gavages tube to determine $L_{50}$. The mortality and survival numbers of rats were recorded for each dose after 96 hours. The data were analyzed statistically by log dose/probit regression line method. The statistical calculations were completed by statistical methods given by Fischer and Yates (1950). The calculated $L_{50}$ of beta-cyfluthrin after 96 hour treatment has been found to be $354.8 \mathrm{mg} / \mathrm{kg}$ body weight (Table-l).

\section{Experimental design}

To assess the effects of beta-cyfluthrin, 5 sets, one acute and four sub-acute sets consisting five and twenty rats respectively, were considered. The control sets were run simultaneously for acute and sub-acute studies having the five and twenty rats respectively. The dosing was done in acute (1 day) and sub-acute $(7,14,21$ and 28 days) treatment after the determination of $\mathrm{LD}_{50}$ (vide supra). The rats in the acute group were given sub-lethal dose of $35.48 \mathrm{mg} / \mathrm{kg}$ body weight (i.e. $1 / 10^{\text {th }}$ of $L D_{50}$ ), while in sub-acute 5.06 , $2.53,1.68$ and $1.27 \mathrm{mg} / \mathrm{kg}$ body weight for 7 , 14,21 and 28 days respectively. Behavioural study has been taken into consideration in both treatment schedules, acute and subacute. Before sacrificing the animals, the body weight has been taken. The control sets were run simultaneously for acute and sub-acute studies having the five and twenty rats respectively.

\section{Biochemical estimations}

The rats were sacrificed under light ether anesthesia after the predetermined time intervals and the brains were dissected out, weighed, homogenized with 0.1 phosphate buffer $(\mathrm{pH} 7.4)$ containing I mM EDTA. The particle free supernatant was obtained by centrifugation and supernatant was used for further biochemical estimation. All the enzyme activities have been expressed in terms of units/mg protein.

Acetylcholinesterase (AChE). (Ellman et al., 1961)

The enzyme activity is measured by following the increase intensity of yellow color produced by the action of thiocholine when it reacts with dithiobisnitrobenzoate ion (DTNB). The intensity was measured at $412 \mathrm{~nm}$ by using a sensitive colorimeter.

Glutathion-S-transferase (GST). (Habig et al., 1974)

Enzyme activity was estimated by determining the rate of formation of glutathione (GSH) and 1 - chloro - 2, 4, dinitrobenzene (CDNB) conjugates. The conjugate was measured colorimetrically at $340 \mathrm{~nm}$.

\section{Adenosine triphosphtase (ATPase) (Seth and} Tangari 1966).

The reaction mixture contained $\mathrm{Mg}^{2+}, \mathrm{Na}^{+}$and $\mathrm{K}^{+}$and reaction was initiated by the addition of ATP and stopped after 10 minutes with TCA. The liberation of inorganic phosphate was estimated at $660 \mathrm{~nm}$ by the method of Fiske and Subbarow (1925). 
Succinic dehydrogenase (Beatty et al., 1966) $\mathrm{SDH}$ catalyzes dehydrogenation of succinate to fumerate. In this reaction, Flavin Adenine Dinucleotide (FAD) converts into dihydrogen flavin adenine dinucleotide $\left(\mathrm{FADH}_{2}\right)$. SDH activity is estimated with the help of INT $\{2-(p-$ iodophenyl)-3-(p-nitrophenyl)-5-phenyl tetrazolium chloride\}, which gives colored formazan, read at $490 \mathrm{~nm}$ spectrophotometrically.

\section{Electrolytes}

The electrolytes were measured both in blood (serum) and brain by ion selective electrode method through analyzer (Perkin and Almer). A comparative method of measurement is utilized. The analyzer measured the potential developed when the sample is pumped through the electrodes. Next standard $A$ is pumped through the electrodes. The difference in the two potentials is related logarithmically to the concentration of sodium and potassium ions in the sample divided by their respective concentrations in the standard solution.

Total proteins (Lowry et al., 1951)

The level of brain total proteins was estimated after Lowry et al. (1951).

A recovery study (45ds) was also done with each set.

\section{Statistical analysis}

The data were analyzed statistically using student ' $t$ ' test and significant difference between the control and treated means were expressed as 'P' values.

\section{Results}

\section{Neurobehavioural alterations}

Moderate to severe signs of toxicity were observed in animals which received different doses of beta-cyfluthrin after $1^{\text {st, }}$ (acute) and $7^{\text {th }}, 14^{\text {th }}$ days (sub-acute) of treatment. Animals showed overt cholinergic signs which included salivation, excitability, mild ataxia, muscle twisting, followed by general tremors and convulsion. The treated groups did not reveal mortality (Table-1).

\section{Neurosomatic index}

The variation in neurosomatic index viz- body weight, brain weight and brain weight to body weight ratio of the animals subjected to different treatments. During the course of present investigations, it was observed the body weights increased $(4.63 \%$ on 1 day and
$37.56 \%, 23.77 \%, 14.66 \%, 5.06 \%$ on $7,14,21$, 28 days respectively). Further brain weights increased $(9.63 \%$ on 1 day and $22.6 \%, 26.7 \%$, $18.18 \%, 16.12 \%$ on $7,14,21,28$ days respectively) and brain weight to body weight ratio has been observed decreased $(7.6 \%$ on 1 day and $7.6 \%, 2.38 \%, 5.1 \%, 5.4 \%, 7.2 \%$ on $7,14,21$ and 28 days respectively) after acute and sub-acute beta-cyfluthrin intoxication when compared to the control animals (Fig. 1).

\section{Neurochemical index}

The acetylcholinesterase (AChE), glutathiones-transferase (GST), adenosine triphosphtase (ATPase) and succinic dehydrogenase (SDH) activities, sodium and potassium activities alongwith total proteins in the brain of albino rat after acute and sub-acute treatments of beta-cyfluthrin. AChE activity of rat brain was found to be inhibited by beta-cyfluthrin. The enzyme activity was maximally inhibited to about $53 \%$ at a dose of $35.48 \mathrm{mg} / \mathrm{kg}$ body weight of beta-cyfluthrin after acute (1 day) treatment; compared to $38 \%, 29 \%, 22 \%$ and $18 \%$ inhibition on $7,14,21$ and 28 days of sub-acute treatment at the doses of 5.06, 2.53, 1.68 and 1.27 respectively. A dose dependent inhibition was found which ranged from $53 \%$ to $18 \%$.. Similar dose-dependent inhibition has been observed in beta-cyfluthrin treated rat brain GST activity. Marked inhibition (47\%) of brain GST has been observed at an acute dose and $41 \%, 36 \%, 34 \%$ and $29 \%$ inhibition on sub-acute doses respectively (vide supra). This inhibition ranged from $47 \%$ to $29 \%$. Further dose-dependent inhibition has been observed in beta-cyfluthrin treated rat brain ATPase activity. Again marked inhibition $(36 \%)$ of brain ATPase has been observed at an acute dose and $33 \%, 25 \%, 22 \%$ and $19 \%$ on sub-acute doses (vide supra). This inhibition ranged from $36 \%$ to $19 \%$. Brain SDH activity maximally inhibited to about $31.6 \%$ at acute dose compared to $31 \%, 21 \%, 11 \%$ and $9 \%$ inhibition on 7, 14, 21 and 28 days of subacute treatment of beta-cyfluthrin respectively, ranging from $31.6 \%$ to $9 \%$ respectively. Further hyponatremia (inhibition range 31 to $20 \%$ ) along with hypokalemia (inhibition range 19 to $14 \%$ ) has also been observed after acute and sub-acute intoxication of beta-cyfluthrin (Fig. 2).

\section{Discussion}

A biomarker is an indicator in a living organism that reflects acute and sub-acute biochemical, physiological and histological changes occurring along a metabolic pathway as a result of exposure to toxicant. Biochemical biomarkers are increasingly used in ecological 
risk assessment of the ecosystem to identify the incidence and effect of xenobiotics. This is because of their potential as rapid early warning signal against potentially damaging effects caused by stressor. In the present study the following biomarkers were evaluated as a battery of tests: Acetylcholinesterase (AChE), Glutathion-s-transferase (GST), Adenosine triphosphtase (ATPsae), and Succinic dehydrogenase (SDH) correlated on the basis of biochemical along with behavioural observations.

\section{Neurosomatic alterations}

The body weight of albino rats has been found to be increased after acute (1d) and sub-acute (7, 14, 21, 28ds) treatment. Body weight reflects the overall state of metabolism and the capability of the organism to maintain its normal growth and development. The increase in body weight may be due to excessive food and water intake and food conversion efficiency of treated groups as compared to controlled ones. The present findings are in accordance to Verma (1996), Sharma (1997) and Kumar (2001) after cypermethrin, hafen 20EC and lambda-cyhalothrin intoxication respectively and are in disagreement with those of Husain et al. (1991) who observed no change in the body weight after deltamethrin intoxication.

The findings in the present study are in accordance to Verma (1996) and Sharma (1997) who also observed increase in brain weight after cypermethrin and hafen 20EC intoxication in albino rats respectively. Further, non-significant reduction in the relative brain weight has been observed after acute and sub-acute treatment and perhaps due to faster increase in the body weight compared to the brain weight. Therefore, relative brain weight decreases. Earlier Ahmed et al. (1989) reported the marked decline in relative weight of brain and assigned extensive neurolysis, glial nodule formation and glial cell proliferation to be responsible for the same. Probably, similar reason can be assigned for decline in ratio (brain weight/body weight) in the present investigation.

\section{Neurobehavioural alterations}

The nervous system is highly susceptible to effect of continuous exposure to xenobiotic substances when gain deliberates or accidental access in the animal body. There is every possibility that the selected experimental compound reveals deleterious neurological disorders particularly in the form of behavioural alterations. Alterations in behaviour under beta-cyfluthrin stress are discussed. The acute and sub-acute doses of Beta-cyfluthrin affect brain central nervous system resulting in spontaneous and delayed behavioural changes. Rats exhibit observable signs of intoxication after 10 minutes of acute dose. The first symptom of toxicity is exhibited about 10 minutes after dose administration as excessive salivation with nausea, rapidly followed by tremors, blinking of eyes, shivering, progressive stretching of hind limbs and immediate thirst for water is observed. All above symptoms that appear are dose dependent. Similar behavioural symptoms have also been observed after sub-acute treatment, however, in lower magnitude from 14 to 21 days and apparently normal after 21 and 28 days. The symptoms of intoxication of Beta-cyfluthrin that appear after both acute and sub-acute treatment are in accordance with Trottier et al. (1980), Lock and Berry (1981), Shivnandappa et al. (1988), Cox (1994), Verma (1996), Saxena (1997), Saxena and Sharma (2000) and Yadav (2002), who observed more or less similar behavioural responses in albino rats following fenitrothion, cypermethrin, cypermethrin, cyfluthrin, cypermethrin, deltamethrin, fenvalerate and confidor ultra pyrethroid intoxication respectively. Repeated exposure to the sublethal doses of an insecticide, signs of poisoning begin but due to their (insecticides) metabolism and detoxification, effects diminish with successive dosing which directly lead to decrease in toxicity. It is because of this reason that the signs of poisoning have been towards decline in the later intervals of experimentation in the present investigation. Again moderate to severe sign of neurobehavioral alterations were observed in rats which received different doses of betacyfluthrin. Rats showed overt cholinergic sign which included salivation, excitability, ataxia, muscle twisting followed by tremors clearly indicate the alterations in behaviour governing enzymes viz-acetyl cholinesterase, cholinesterase, and energy linked enzymes viz-adenosine triphosphatase and succinic dehydrogenase which has been observed in the present study (vide infra).

\section{Neurochemical alterations}

AChE is an enzyme that is essential for the normal functioning of the central and peripheral nervous system (Hart, 1993) and is widely distributed in the neural and non-neural tissues (Fribroulet et al., 1990). The structural studies of AChE indicates the presence of different binding sites, one, a selective aromatic cation binding site; two, esteratic site and three, peripheral anionic substrate binding 
site. The ACh molecule interacts both with esteratic and anionic sites. The esteratic site includes imidazole and hydroxyl side chains of histidine and serine. Within the anionic site, ester group represents 6 to 9 active negative charges. Site 2 (esteratic) and site-3 (anionic) participate in catalytic reaction and binding of choline occurs at these two sites. Further, active site is rich in aromatic content which creates a hydrophobic area which is different from the binding site of $\mathrm{ACh}$ as revealed by spectroscopy (Sussman et al. 1991). It is well known that compounds like organophosphates bind to the hydrophobic area and cause inhibition in AChE activity levels. This aromatic hydrophobic lining probably permits the initial absorption of ACh to low affinity sites, followed by the respective diffusion to active sites, and release of the product choline after reaction. This aromatic surface on AChE, in turn, will provide the basis for binding a xenobiotic substance with substrate Ach (Sussman et al., 1991). To some extent the synthetic pyrethroids reveal hydrophobicity in vivo which probably is its key mechanism to interact at the aromatic surface of AChE with the result of which ACh binding space gets reduced and in turn lead to the inhibition in AChE activity (Rao and Rao; 1995) [Fig. 3]. In the present investigation there is every possibility that beta-cyfluthrin being a type-II pyrethroid may interact at the same region (vide supra) leading to the inhibition of AChE activity. Further the decrease in brain AChE activity by beta-cyfluthrin may be attributed to the fact that pyrethroids potentially inhibit neurotransmitter metabolizing enzymes in the brain, which are essential for maintaining the balance of neurotransmitter like ACh in the brain; leads to decrease in the activity of AChE levels (Rao and Rao, 1995). It is likely that beta-cyfluthrin (type-II pyrethoid) exerts an imbalance in neuro-chemical homeostasis resulting AChE inhibition Trottier et al. (1980), Bandyopadhyay (1982), Latuszynska et al; (2001) and Timur et al; (2003) also observed inhibition in the activity of brain AChE. Again, moderate to severe sign of neurobehavioural alterations were observed in rats which received different doses of beta-cyfluthrin., rats showed overt cholinergic sign which included salivation, excitability, ataxia, muscles twisting followed by tremors, clearly indicate the impairment in the activity of acetylcholinesterase in brain of albino rats in the present investigation and in accordance to Eriksson et al. (1993) and Ahlbom et al. (1994) after intoxication of bioallethrin in rats respectively.
Brain GST activity was studied at different doses, along with AChE activity. Quantitation in the present studies has shown that brains do possess glutathione-stransferase (GST) though in a very little amount, yet participates in detoxification. The GST catalyzes the nucleophilic adding of the thiol of reduced glutathione to a variety of electrophilies, in addition, GST bind with varying affinities a variety of hydrophobic compounds such as polycyclic aromatic hydrocarbons and other xenobiotics as pyrethroids etc. GST conjugate numerous xenobiotic with glutathione. Therefore the endogenous availability of glutathione at the target site might be a limiting factor for GST catalyzed conjugation and thereby influence the protective function of the enzyme, but any alterations in the activity of GST can lead to alterations in activation-detoxification balance present in tissues to detoxify potential toxic agents. (Rushmore and Pickett 1993).

Brain GST activity has been found to be decreased after acute and sub-acute treatment of beta-cyfluthrin is in accordance to Rushmore and Pickett (1993), Timur et al; (2003), and Johri et al; (2006), who observed decrease in brain GST activity after dexamethasome, malathion and deltamethrin treatment in rats, respectively. The observed GST inhibition clearly indicate the affinity of this enzyme towards the hydrophobic compounds like pyrethroids, leading to the decrease in the activity of GST in brain in the present investigation. The decrease in brain GST activity after acute and sub-acute treatment of beta-cyfluthrin may possibly be attributed to the compensatory biotransformation activity in the brain of rats triggered by action of pesticide in question. A decrease in brain GST activity may be because of action of pesticide, which alters detoxification metabolism and gains support by the work of Timur et al. (2003) and Johri et al. (2006) who also observed decrease activity of GST in the brain of rats after malathion and deltamethrin toxicity. Furthermore, in subacute treatment, inhibition in brain GST activity clearly indicates some adaptive changes could counteract beta-cyfluthrin induced alterations in brain biochemistry specially in detoxification mechanism.

Adenosine tri-phosphtase (ATPase) a membrane associated enzyme synthesized in inner mitochondrial matrix; catalyze the decomposition of Adenosine triphosphate (ATP) into Adenosine di-phosphate (ADP) with a free phosphate ion (dephosphorylation), which ultimately leads to release of energy. 
Adenosine tri-phosphate

\section{$\longrightarrow$}

Adenosine di-phosphate (ADP) + Inorganic phosphate

Adenosine tri-phosphatase is essential for maintenance of membrane potential in excitable cells and it is involved in diverse physiological functions vizneurotransmitter release and their uptake (Anbrasi et al. 2005). Brain total ATPase activity has been found to be significantly decreased after acute and sub-acute treatments of beta-cyfluthrin is in accordance to Pentyala and Chetty (1993), Vani et al. (2000), and Rao et al. (2003), who observed decrease in brain Adenosine tri-phosphatase activity after cypermethrin, propoxur, thiobencarb, fluoride and lead exposure in rats respectively. The brain which has important energy needs, an adequate and timely production of ATP by brain cells is of cardinal importance to support the major energetic cost of the rapid processing of informations, is highly vulnerable to energetic dysfunctions. It is likely that mitochondrial energetic system must have been disturbed out due to the movement of molecules of beta-cyfluthrin within mitochondria, after its access through the meninges. Furthermore the normal functioning of membrane associated enzymes are dependent on their lipid environment and any perturbation of lipid environment in the vicinity of xenobiotics, could result in significant alterations in membrane enzymes (ATPase) after the entry of beta-cyfluthrin through the brain meninges and gains support by Vani et al. (2000), Rao et al. (2003) and Pushpakiran et al. (2005).

Succinic dehydrogenase (SDH), an important enzyme of the Kreb's cycle of mitochondria is encoded on the fragment of chromosome 1. In fact, SDH contains three different kinds of iron-sulphur clusters, embedded in the inner mitochondrial membrane. In the mitochondrial membrane, $\mathrm{SDH}$ unite with ubiquinone from succinicubiquinone reductase, the so-called complex II of the respiratory chain. SDH is involved in Kreb's cycle and catalyzes succinic to fumarate. Along with brain cells, this reaction is important in nearly all mammalian cells (Stachowska et al. 2000).

The level of brain succinic dehydrogenase activity has been found to be decreased after acute and sub-acute treatments of beta-cyfluthrin and in recovery studies is in accordance to Brouillet et al. (1998), Zatta et al. (2000) and Stachowska et al. (2000), who observed decreased brain $\mathrm{SDH}$ activity after nitropropionic acid, aluminium and fluoride exposure in rats respectively. The observed decrease in brain succinic dehydrogenase activity may be an outcome of compensatory physiochemical activity of brain viz-impaired oxidative and energy metabolism, which is adjudged on the basis of altered mitochondrial oxidative metabolism in brain cells. It is noteworthy that xenobiotics like fluoride inhibit the cellular energy production (Stachowska et al., 2000 and Zatta et al. 2000). They pass through the inner mitochondrial membrane and inhibit the enzyme activity of the respiratory chain. Betacyfluthrin, a type-Il pyrethroid, having fluoride molecule in its chemical structure [Fig.3], revealed inhibition in energy metabolism by inhibiting the succinic-dehydrogenase activity of brain cells by the fluoride of beta-cyfluthrin and gains support by Stachowska et al. (2000). The brain total proteins have been observed to be decreased (hypoproteinaemia) non-significantly after acute (1d) and subacute $(7,14,21$ and 28 ds) treatment of betacyfluthrin, is in accordance to Ahmed et al (1986), and Timur et al. (2003), who also observed inhibition in rat brain total proteins after administration of dieldrin and malathion respectively. The decrease in brain total proteins may probably be due to the inhibition in protein synthesis and defective enzyme system as all enzymes are proteins revealed by Ahmed et al. (1986).

Electrolytes are the salts that are present in mammalian body. Electrolytic disorders can produce numerous clinical manifestations in a mammalian body especially in brain. Too much or too little electrolytes content in brain can cause malfunctioning of the tissue. Hypnoatremia, along with hypokalemia has been observed in brain after acute (1d) and sub-acute (7, 14, 21, 28ds) beta-cyfluthrin intoxication are in accordance to Ghiasuddin and Soderlund (1984), Lian et al. (1991), Rao (1997), Trainer et al. (1997), Narashi (1998), and Chaudhary (2004) and Anabarasi et al. (2005) after intoxication of permethrin, deltamethrin, DDAVP, permethrin, cypermethrin, hafen20EC and $\lambda$-cyhalothrin in albino rats respectively. A significant decrease in brain sodium has been observed after betacyfluthrin intoxication, might be attributed to the plugging of sodium channels at their gate in the brain (Narashi et. al. 1998) and the observed toxic neurobehavioral alterations like tremors, convulsions, thirst, and ataxia that appeared in the treated rats in the present investigation. Hyponatremia may lead to 
alterations in the level of consciousness, tremor, confusion and lethargyness along with ataxia and depression. The present investigation is affirmated by the findings of Ricard and Brodie (1985), Forsha and Ray (1985) and Saxena and Sharma (2000) after intoxication of deltamethrin, cismethrin and hafen 20EC in rats respectively. Further a significant decrease in brain potassium has been observed after beta-cyfluthrin intoxication. This might be due to the direct plugging of potassium channels (Narahashi et al. 1998), because the mechanism of action of pyrethroids suggests that beta-cyfluthrin, a type-II pyrethroid molecules directly plug the potassium channels in the brain and again the observed toxic symptoms in from of behavioural alteration viz-tremors, convulsions indicates the hypokalemia in albino rats under stress of beta-cyfluthrin. There is minimal change in every parameter either it is behavioural, biochemical after 45ds (recovery period) which reveals detoxification in albino rats.

However, on the basis of above mentioned account it can be interpreted that the selected cyno group derivative (betacyfluthrin, type-Il pyrethroid) has a potential to induce central nervous system alterations along with biochemical irregularities in brain which forces its regulated application in the ecosystem else the chemical may prove its worth as a future neurotoxin like the conventional $\mathrm{II}^{\text {nd }}$ and $\mathrm{III}^{\text {rd }}$ generation pesticides of yester years. It is quite obvious that betacyfluthrin application needs proper scientific management in order to save the tertiary consumers as well as maintaining its original status of being non toxic and easily detoxifiable pyrethroid. The brain biochemical, behavioural and somatic alterations are suggestive of stress which was induced by acute and sub-acute dosing schedule per O.S.

\section{References}

Ahlbom J, Fredriksson A and Eriksson P, 1994. Neonatal exposure to a type-I pyrethroid (bioallethrin) induces dose-response changes in brain muscarinic receptors and behaviour in neonatal and adult mice. Brain Research, 645 : 318-324.

Ahmed N, Gupta PK and George KC, 1989. Sub acute toxicity of cypermethrin in rats. Journal of Environmental Biology, 10 (3) : 309-317.

Anbarasi K, Vani G, Balakrishna K and Devi CS, 2005. Effect of bacoside $A$ on membrane bound ATPase in the brain of rats exposed to cigarette smoke. Journal of Biochemical Molecular Toxicology, 19: 59-65.

Bandyopadhyay R, 1982. Inhibition of acetylcholine esterase by permethrin and its reversion by acetylthiocholine. Indian Journal of Experimental Biology, 20 : 489-491.

Beatty $\mathrm{CH}$, Basinger GM, Dully CC and Bocek RM, 1966. Comparison of red and white voluntary skeletal muscles of several species of primates. Journal of Histochemistry and Cytochemistry, 14 (8): 590-600.

Brouillet E, Guyot MC, Mitoux V, Altairac S, Francoise C, Stephane P and Phillippe AH, 1998. Partial inhibition of brain Succinate dehydrogenase by 3-nitropropionic acid is sufficient to initiate striatal degeneration in rat. Journal of Neurochemistry, 70: 794-805.

Chaudhary A, 2004. Effect of lambda cyhalothrin and piperonyl butoxide on the hepato-renal functions of male albino rat. Ph.D. Thesis. Dr. B.R. A. Univ. Agra.

Cox C, 1994. Cyfluthrin. Journal of Pesticide Reformation, 14 (2) : 28-34.

Ellman GL, Courney KD, Andres $\mathrm{V}$ and Featherstone RM, 1961. A new and rapid colorimetric determination of acetyl cholinesterase activity. Biochemical Pharmacology, 7 : 88-95.

Eriksson, P., U. Johansson, J. Ahlbom and A. Fredriksson. 1993. Neonatal exposure to DDT induces increased susceptibility to pyrethroid (bioallethrin) exposure at adult age-changes in cholinergic muscarinic receptor and behavioural variables. Toxicology, $77: 21-30$.

Fisher and Yates, 1950. Statistical Tables for Biological, Agriculture and Medical Research, Longman VI edition, $\mathrm{X}+146 \mathrm{pp}$.

Fiske $\mathrm{CH}$ and Subbarao Y, 1925. The colorimetric determination of phosphatase, Journal of Biological Chemistry, 66: $375-400$.

Forshaw PJ and Ray DE, 1985. The effect of two pyrethroids, cismethrin and deltamethrin on skeletal muscle and the trigeminal reflex system in the rat. Pesticide Biochemistry and Physiology, 25 : 143151.

Friboulet A, Reiger F, Goudou D, Amitai G and Taylor P, 1990. Interaction of an organophosphate with a peripheral site on acetylcholinesterase. Biochemistry, 29: 914-920.

Ghiasuddin SM and Soderlund DM, 1984. Hydrolysis of pyrethroid insecticides by soluble mouse brain esterases. Toxicology and Applied Pharmacology, 74 : 390-396.

Habig WH, Michael JP and William BJ, 1974. Glutathione-S-transferase. The first enzymatic step 
in mercapturic acid formation. Journal of Biological Chemistry, 249(22): 7130-7139.

Hart ADM, 1993. Relationships between behavior and the inhibition of acetylcholinesterase in birds exposed to organophosphorus pesticides. Environmental Toxicology and Chemistry, 12: 321 336.

Hussain R, Gupta A, Khanna VK and Seth PK, 1991. Neurotoxicological effects of a pyrethroid formulation, fenvalerate in rats. Research Communication. Clinical Pathology and Pharmacology, 73 (1) : 111-114.

Johri A, Yadav S, Singh RL, Dhawan A, Ali M and Parmar D, 2006. Long lasting effect of prenatal exposure of deltamethrin on cerebral and hepatic cytochrome p450 and behavioral activity of rat offspring. European Journal of Pharmacology, 544(1-3): 58-68.

Kumar R, 2001. Biochemical alteration in rat brain following type II pyrethroid intoxication. M.Phil. Dissertation. Dr. B.R.A. Univ., Agra.

Latuszynska J, Luty S, Raszewski G, Rodaki MT, Prezebirowska D, Przylepa E and Maj AH, 2001. Neurotoxic effects of dermally applied chlorpyrifos and cypermethrin in wistar rats. Annals of Agriculture and Environmental Medicine, 8 : 163170.

Lian Y, Shapiro Jl and Chan L, 1991. Study of brain electrolytes and organic osmolytes during correction of chronic hyponatremia. Journal of Clinical Investigation, 88: 303-309.

Lock EA and Berry PN, 1981. Biochemical changes in the rat cerebellum following cypermethrin administration. Toxicology and Applied Pharmacology, 59 (3) : 508-514.

Lowry $\mathrm{AH}$, Rosenbrough NJ, Parr AL and Randall RJ, 1951. Journal of Biological Chemistry, 193: 265-275.

Narahashi T, Ginsburg KS, Nagata K and Song JH, 1998. Ion channels as targets for insecticides. Neurotoxicology, 19 (4-5): 581-90.

Pentyala SN and Chetty CS 1993. Comparative changes in AChE and ATPase activities in neonate and adult rat brains under thiobencrab stress. Journal of Applied Toxicology, 13(1): 39-42.

Pushpakiran G, Mahalakshmi K, Viswanathan P, and Anuradha CV, 2005. Taurine prevents ethanol induced alterations in lipids and ATPase in rat tissues. Pharma Representative, 57: 578-587.

Rao GV and Rao KS, 1997. Modulation of potassium across synaptosomes of rat brain by synthetic pyrethroids. Journal of Neurological Science, 147 (2): 127-33.
Rao GV and Rao KSJ, 1995. Modification in acetyl cholinesterase of rat brain by Pyrethroids in vibo and an vitro kinetic study. Journal of Neurochemistry, 65 : 2259-2266.

Rao P, Butler J, Kumar BK and Rajanna B, 2003. In vitro effect of lead on $\mathrm{Na}^{+}, \mathrm{K}^{+}$-ATPase activity in different regions of rat brain. Drug Chemistry and Toxicology, 26 (2): 117-124.

Rickard J, and Brodie ME, 1985. Correlation of blood and brain levels of the neurotoxic pyrethroid deltamethrin with the onset of symptoms in rats. Pesticide Biochemistry and Physiology, 23 : 143156.

Rushmore TM and Pickett CB, 1993. Gluatathione S-transferases, structure, regulation and therapeutic implications. Journal of Biological Chemistry, 268 (16) : 11475-11470.

Saxena M, 1997. Effect of deltamethrin on brain biochemistry of Rattus norvegicus. M. Phil. Dissertation. Dr. B.R.A. Univ., Agra.

Saxena PN and Sharma DC, 2000. Effect of synthetic pyrethroid on behaviour Pattern in Rattus norvegicus. Proceedings of National Academy of Sciences, India, 70 (B) I: 41-43.

Seth PK and Tangari RK, 1966. Biochemical methods of newer salicylic acid cogenesis, Journal of Pharmacology, 18: 831-833.

Sharma DC, 1997. Brain biochemical changes of albino rat after hafen-20EC intoxication. M.Phil. Dissertation. Dr. B.R.A. Univ., Agra.

Shivanandappa T, Joseph $\mathrm{P}$ and Krishna Kumari MK, 1988. Response of blood and brain cholinesterase to dermal exposure of bromophos in the rat. Toxicology, 48 : 199-208.

Stachowska E, Bober J, Chlubek D and Machoy Z, 2000. Number of Fluoride ions binding to Succinate dehydrogenase during mixed inhibition. Fluoride, 33 (3): 115-120.

Sussman JL, Harel M, Frolow F, Oefner C, Goldman A and Silman I, 1991. Atomic structure of Acetylcholinesterase from Torpedo californica: a protypic acetylcholine binding protein. Science, 252 : 872-879.

Timur S, Onal S, Karabay NU, Sayim F and Zihhioglu $F$, 2003. In vivo effects of malathion on glutathione-s-transferase and acetylcholinesterase activity in various times of neonatal rats. Turkish Journal of Zoology, 27 : 247-252.

Trainer VL, Mcphee JC, Bochan HB, Baker C, Schever T, Babin D, Domoute JP, Gvedin D and Catterall WA, 1997. High affinity binding of pyrethroids to the $\alpha$-subunit of brain sodium channels. Molecular Pharmacology, 51 : 651-657. 
Trottier B, Fraser AR, Planet G and Ecobichon DJ, 1980. Sub acute toxicity of technical of technical fenitrothion in male rats. Toxicology, 17: 29-38.

Vani M and Reddy KP, 2000. Effects of Fluoride accumulation on some enzymes of brain and gastrocnemius muscle of mice. Fluoride, 33 (1): 17 26.

Verma R, 1996. Effect of cypermethrin on certain brain biochemical parameters in albino rat. M.Phil. Dissertation. Dr. B.R.A. Univ, Agra.
Yadav E, 2002. Effect of confidor ultra, a synthetic pyrethroid on haematology of albino rat. M.Phil. Diss. Dr. B.R. A. Univ., Agra.

Zatta P, Lain E and Cagnolini C, 2000. Effects of aluminum on activity of Krebs cycle enzymes and glutamate dehydrogenase in rat brain homogenate. European Journal of Biochemistry, 267: 3049-3055.

Figures and Table follow. 


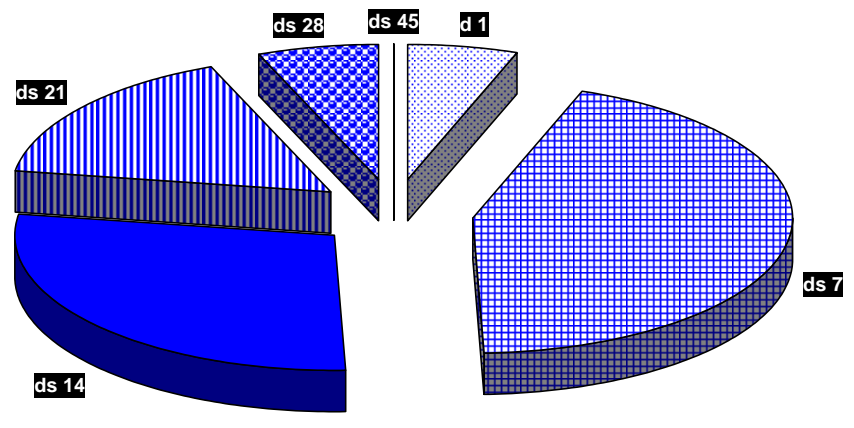

BODY WEIGHT

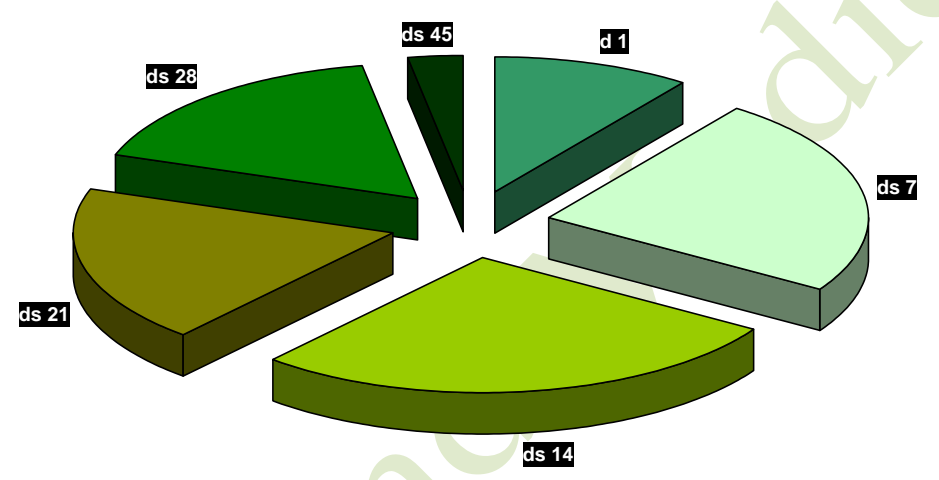

BRAIN WEIGHT
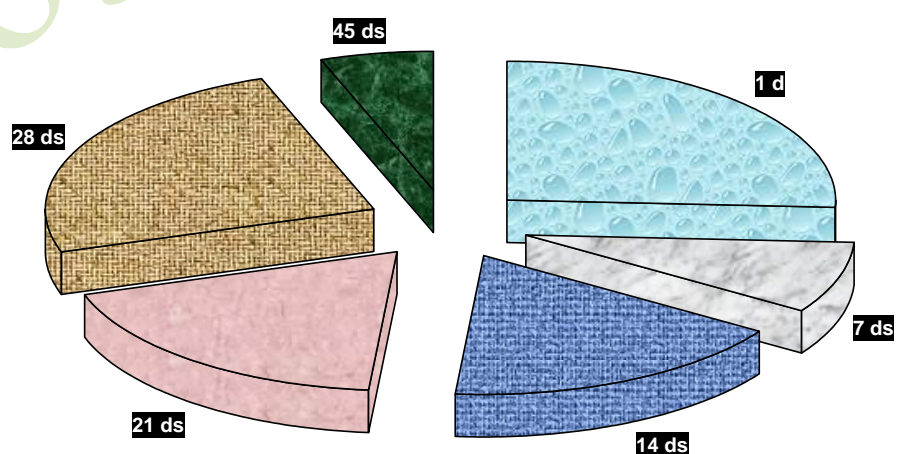

BRAIN WEIGHT-BODY WEIGHT RATIO

Fig. 1: \% change in body weight, brain weight and brain weight-body weight ratio after treatment of beta-cyfluthrin 
Fig. 2: \% change in AChE, GST, ATPase, $\mathrm{SDH}, \mathrm{Na}^{+}$and $\mathrm{K}^{+}$after treatment of beta-cyfluthrin
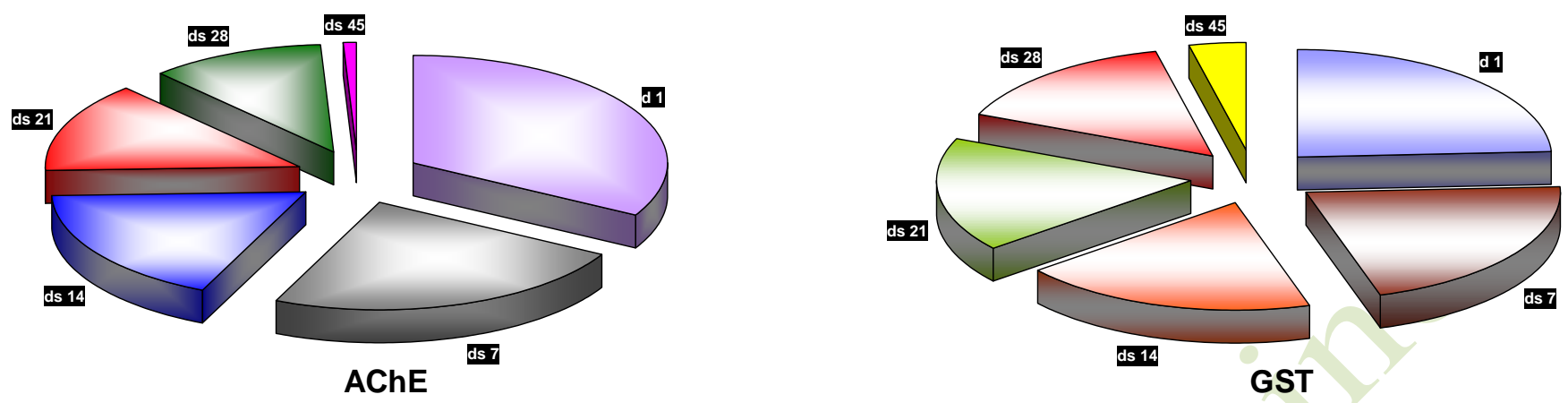
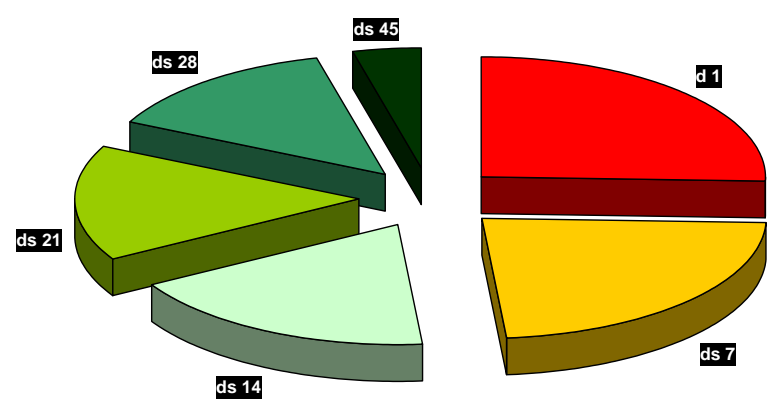

ATPase

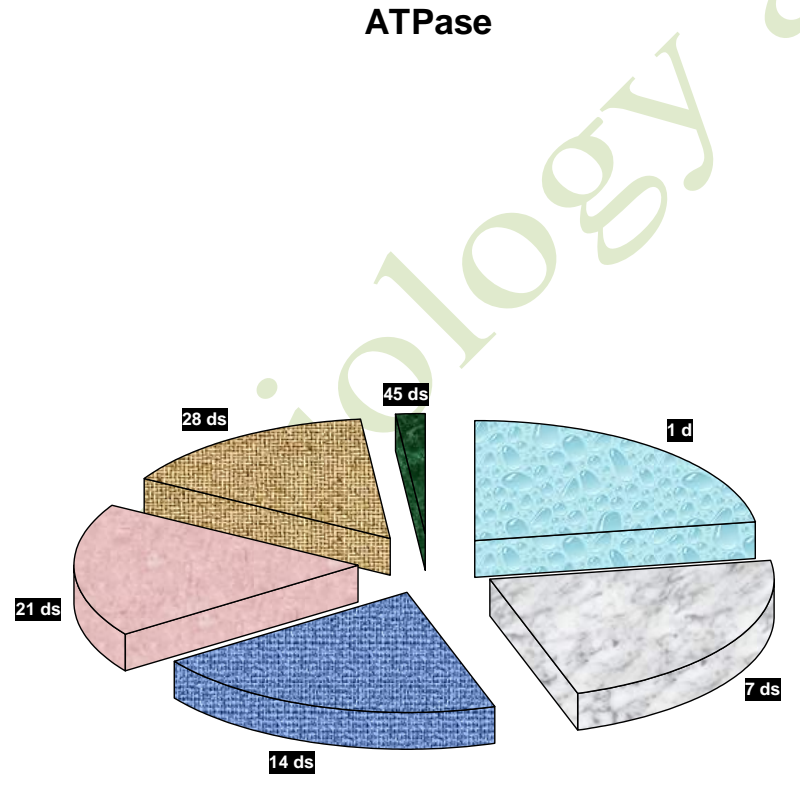

$\mathrm{Na}^{+}$

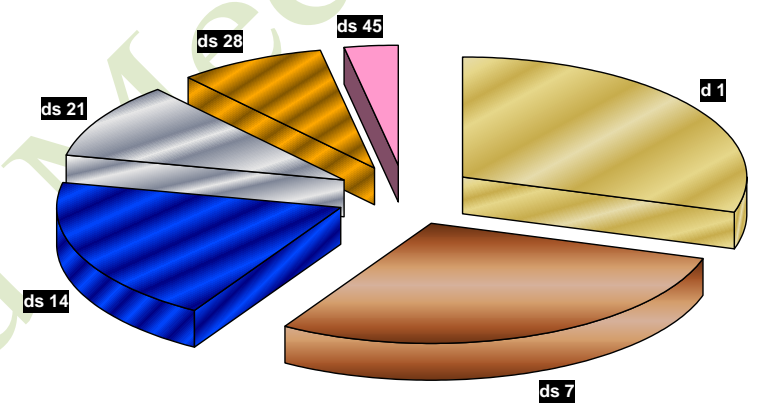

SDH

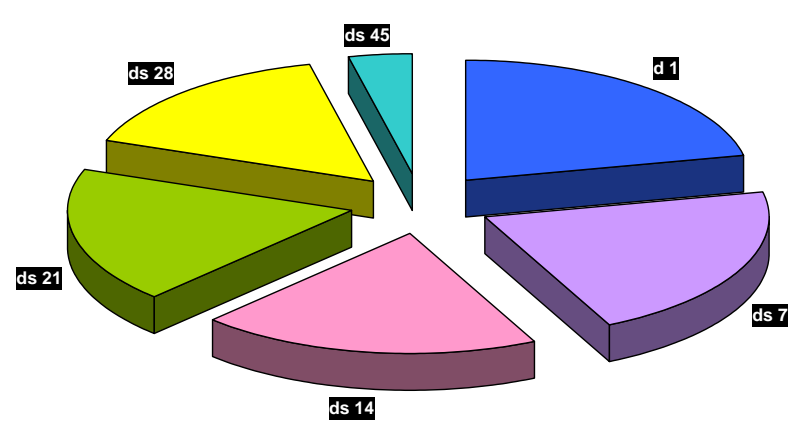

$\mathrm{K}^{+}$ 


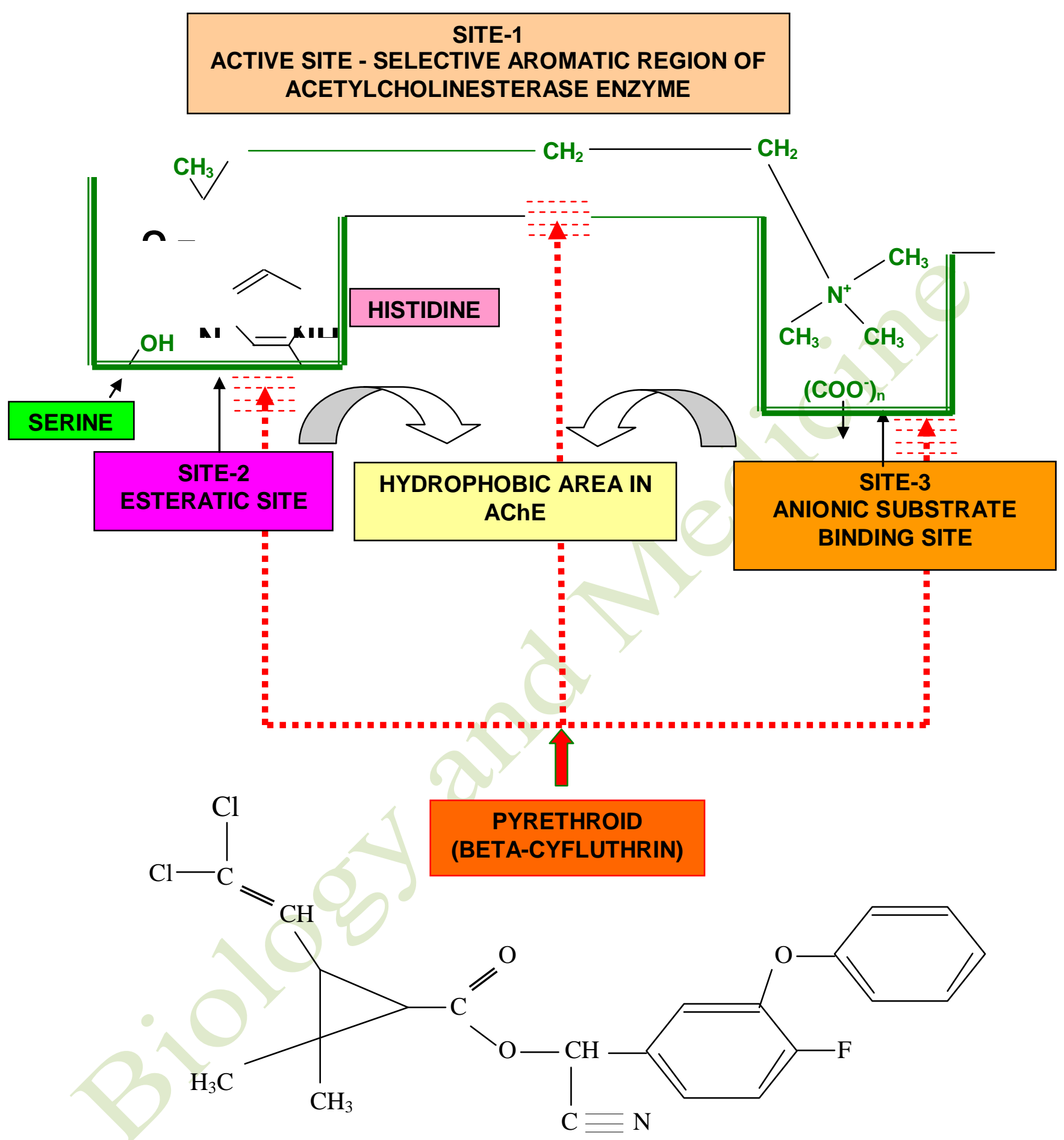

FIG. 3- SCHEMATIC REPRESENTATION OF BINDING SITES OF ACETYLCHOLINESTERASE ENZYME (AChE) AND BINDING POSITIONS OF BETA-CYFLUTHRIN 
Table 1: Neurobehavioural changes in Rattus norvegicus after beta-cyfluthrin intoxication.

\begin{tabular}{|c|c|c|c|c|c|c|c|c|c|c|c|c|c|c|c|c|c|}
\hline 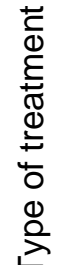 & \multicolumn{2}{|c|}{ 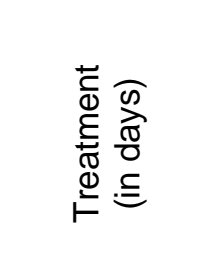 } & 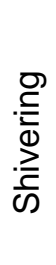 & 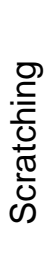 & 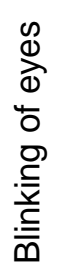 & 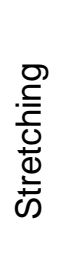 & 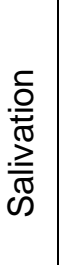 & 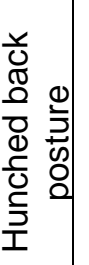 & 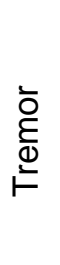 & 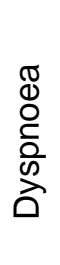 & $\begin{array}{l}\frac{\pi}{x} \\
\underset{\pi}{\frac{\pi}{<}}\end{array}$ & 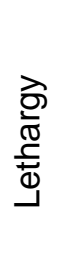 & 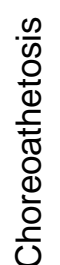 & $\begin{array}{l}\stackrel{\vec{n}}{\vec{E}} \\
\stackrel{F}{F}\end{array}$ & 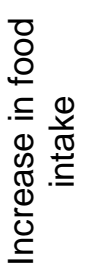 & 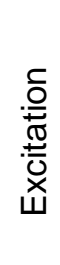 & 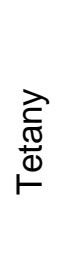 \\
\hline \multirow{2}{*}{$\frac{\text { 影 }}{\frac{3}{2}}$} & \multirow{2}{*}{$\tau$} & $\infty 0$ & 成 & 8 & $\#$ & $\theta$ & $\#$ & $\theta$ & $\neq$ & $\square$ & $\square$ & 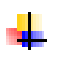 & $\#$ & $\#$ & 8 & $\theta$ & $\theta$ \\
\hline & & O & 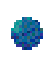 & $\square$ & 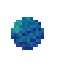 & 8 & ? & $\square$ & 图 & 圆 & $\#$ & 圆 & 圆 & $\#$ & $\#$ & 8 & 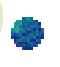 \\
\hline \multirow{8}{*}{ 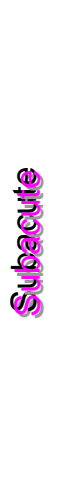 } & \multirow{2}{*}{$\Lambda$} & の & 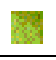 & 8 & $\#$ & 8 & $\#$ & 8 & 8 & $\#$ & $\#$ & 8 & 8 & 8 & $\square$ & 8 & 8 \\
\hline & & 0 & 9 & $\square$ & 9 & 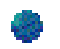 & 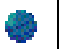 & $\neq$ & 9 & 圈 & $\varphi$ & $\theta$ & $\varphi$ & $\varnothing$ & & $\theta$ & $\varphi$ \\
\hline & \multirow{2}{*}{$\stackrel{\Xi}{\square}$} & $\infty 0$ & 8 & $\theta$ & 8 & 8 & 8 & 8 & 8 & 国 & 8 & 8 & 8 & 8 & $\#$ & $\theta$ & $\theta$ \\
\hline & & 00 & 8 & 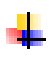 & $\theta$ & $\theta$ & $\theta$ & $\neq$ & $\theta$ & $\theta$ & $\theta$ & 8 & 8 & 8 & 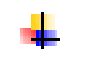 & 8 & 8 \\
\hline & \multirow{2}{*}{$\bar{N}$} & $\infty 0$ & $\#$ & $\theta$ & 8 & 8 & $\theta$ & $\theta$ & $\varphi$ & $\square$ & 8 & $\#$ & 8 & $\#$ & \# & $\#$ & 8 \\
\hline & & 00 & $\theta$ & $\square$ & $\theta$ & $\theta$ & 8 & $\square$ & 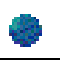 & $\neq$ & $\neq$ & 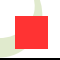 & $\neq$ & $\theta$ & $\square$ & 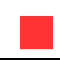 & 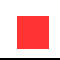 \\
\hline & \multirow{2}{*}{$\stackrel{\infty}{\sim}$} & $\infty 0$ & $\#$ & 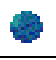 & 8 & 8 & 8 & $\theta$ & 8 & $\square$ & in & $\#$ & 国 & $\#$ & $\#$ & $\#$ & 8 \\
\hline & & O & $\theta$ & $\#$ & $\theta$ & $\theta$ & $\theta$ & 圆 & $\theta$ & 8 & II & $\neq$ & 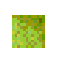 & $\theta$ & 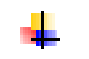 & \# & $\neq$ \\
\hline \multirow{2}{*}{ 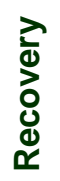 } & \multirow{2}{*}{ ᄂீ } & ט D & $\theta$ & $\theta$ & $\theta$ & 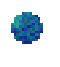 & $\theta$ & $\theta$ & 9 & $\theta$ & $\theta$ & $\theta$ & $\theta$ & $\theta$ & $\theta$ & 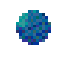 & $\theta$ \\
\hline & & ט D & $\theta$ & $\theta$ & 8 & $\theta$ & 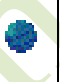 & 8 & 9 & $\theta$ & 8 & $\theta$ & $\theta$ & $\theta$ & $\theta$ & 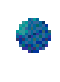 & 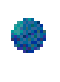 \\
\hline
\end{tabular}

No change

Mild

Moderate

Severe
SC- Spontaneous changes

DC- Delayed changes 